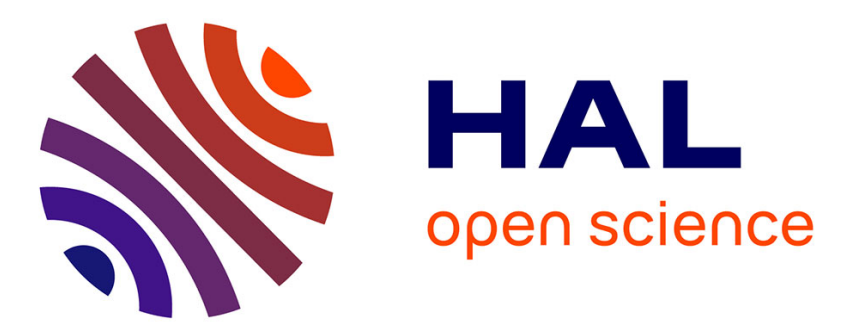

\title{
Soluble fullerene derivative in liquid crystal: polymer composites and their impact on photorefractive grating efficiency and resolution
}

\author{
Alexey Denisov, Jean-Louis de Bougrenet de La Tocnaye
}

\section{- To cite this version:}

Alexey Denisov, Jean-Louis de Bougrenet de La Tocnaye. Soluble fullerene derivative in liquid crystal: polymer composites and their impact on photorefractive grating efficiency and resolution. Applied optics, 2009, 48 (10), pp.1926-1931. hal-00460290

\author{
HAL Id: hal-00460290 \\ https://hal.science/hal-00460290
}

Submitted on 26 Feb 2010

HAL is a multi-disciplinary open access archive for the deposit and dissemination of scientific research documents, whether they are published or not. The documents may come from teaching and research institutions in France or abroad, or from public or private research centers.
L'archive ouverte pluridisciplinaire $\mathbf{H A L}$, est destinée au dépôt et à la diffusion de documents scientifiques de niveau recherche, publiés ou non, émanant des établissements d'enseignement et de recherche français ou étrangers, des laboratoires publics ou privés. 


\title{
Soluble fullerene derivative in liquid crystal: polymer composites and their impact on photorefractive grating efficiency and resolution
}

\author{
Alexey Denisov* and Jean-Louis de Bougrenet de la Tocnaye \\ Optics Department, TELECOM Bretagne, CS 83818, 29238 Brest Cedex, France \\ ${ }^{*}$ Corresponding author: alexey.denisov@enst-bretagne.fr
}

Received 21 January 2009; revised 11 March 2009; accepted 11 March 2009; posted 13 March 2009 (Doc. ID 106593); published 26 March 2009

\begin{abstract}
By using soluble fullerene derivative [60]PCBM, we improved photorefractive efficiency in polymerliquid crystal composites in comparison to previous works on similar materials. We show the effect of polymer network results in resolution and bandwidth improvements compared to pure liquid crystals. This is explained by the introduction of a charge trapping mechanism, providing a memory effect for the composite. Based on this effect, we propose an approach for designing composites with higher grating efficiency and resolution. (c) 2009 Optical Society of America

OCIS codes: $\quad 190.5330,160.5320,160.3918,050.2770,160.3710,160.5470$.
\end{abstract}

\section{Introduction}

Since the discovery of the photorefractive effect in liquid crystals (LC) in 1994 [1, 2 ], constant advances have been achieved in their performance [3- $\underline{3}]$. According to past studies, the photorefractive effect in LC is due to combined effects of the photo-induced ionic generation of a space-charge field, as shown in [2] and the space-charge field resulting from dc anisotropic conductivity (Carr-Helfrich effect) [3]. Improvements were obtained mainly by reducing the intensity of the writing beams to a few milliwatts per square centimeter and the applied electric field to less than $0.1 \mathrm{~V} / \mu \mathrm{m}[7,8]$. High-index modulation was obtained only for grating periods $\Lambda_{x}$ larger than the LC cell thickness $d$. As shown in [3], the charge flow mechanism of the grating formation results in an index modulation maximum at $\Lambda_{x} \approx 2 d$ and sharply decreases for smaller grating periods $[3,8]$. Subsequent work $[5,6]$ indicated that the photorefractive gratings in LC have a surface origin, which explains a sharp decrease in diffraction efficiency at small grating periods. Photorefractive LC resolution can

0003-6935/09/101926-06\$15.00/0

(C) 2009 Optical Society of America be improved by combining them with polymers [9-15]. Pioneering work was performed in polymer dispersed liquid crystals (PDLC) [9,11] whereas, later, mesogenic polymer was propose $\bar{d}[13-15]$, making samples transparent and requiring lower applied voltages. The main interest of LC polymer composites is the capability of a decoupling between cell thickness and grating period, which is not possible for the surface-driven photorefractive effect in LC. In this frame, one needs to distinguish between PDLC or polymer stabilized liquid crystals (PSLC with a polymer concentration $<15 \%$ ) doped with charge generators (TNF, fullerene) and containing a conducting polymer matrix, such as polymethyl methacrylate (PMMA) doped with $N$-ethylcarbazole (ECZ) for hole transport [11,12] and PDLC (or PSLC) doped only with charge generators $[9,10,14,15]$. The first type of PDLC has a different mechanism of charge generation than the one for low molecular mass nematics $[2,4]$. The absence of photoconductivity due to the absence of ECZ reported in [12] and the higher applied voltages of $15-50 \mathrm{~V} / \mu \mathrm{m}$ that are required show that the polymer participates in the charge separation phenomenon. In the second PDLC type, polymer serves only as an inert matrix, whereas the charge generation mechanism is due 
to the interaction between low molecular mass nematics and the charge generating dopants $[2,4]$.

Here we concentrate on the PSLC with an inert polymer matrix, which belongs to the second type of polymer-LC composites. We show diffraction efficiency improvements at small grating periods in comparison to previous works on similar media $[9,10]$, as well as diffraction efficiency improvements compared to pure LC, highlighting the role of the polymer network. The diffraction efficiency has been shown in previous work to depend on the polymer concentration $[9,10]$ and the maximum efficiency was observed in the PSLC case at low polymer concentrations (5-15\%) in contrast to PDLC (having LC droplets encapsulated in the host polymer, for concentrations greater than $30 \%)$. The dependence on the dopant concentration (fullerene $\mathrm{C}_{60}$ ) was not investigated because of its low maximum solubility in PSLC composite: $0.05 \%$. We observed that functionalized fullerene phenyl- $\mathrm{C}_{61}$ butyric acid methyl ester ([60]PCBM; see Fig. 1) has a greater solubility in PSLC used in $[9,10]$. This functionalized fullerene was developed for greater solubility in organic solvents, while preserving the electronic and optical properties of the parent compound $\mathrm{C}_{60}$, with the target of organic electronic devices [16]. Increase in charge generator concentration results, in our case, in an increase in diffraction efficiency, compared to $[\underline{9}, \underline{10}]$, especially at small grating periods.

\section{Material Description}

The investigations were performed on a PSLC doped with [60]PCBM. The PSLC is made up of a PMMA and nematic mixture E7. The mixture provided a direct comparison with $[9,10]$, where a similar mixture

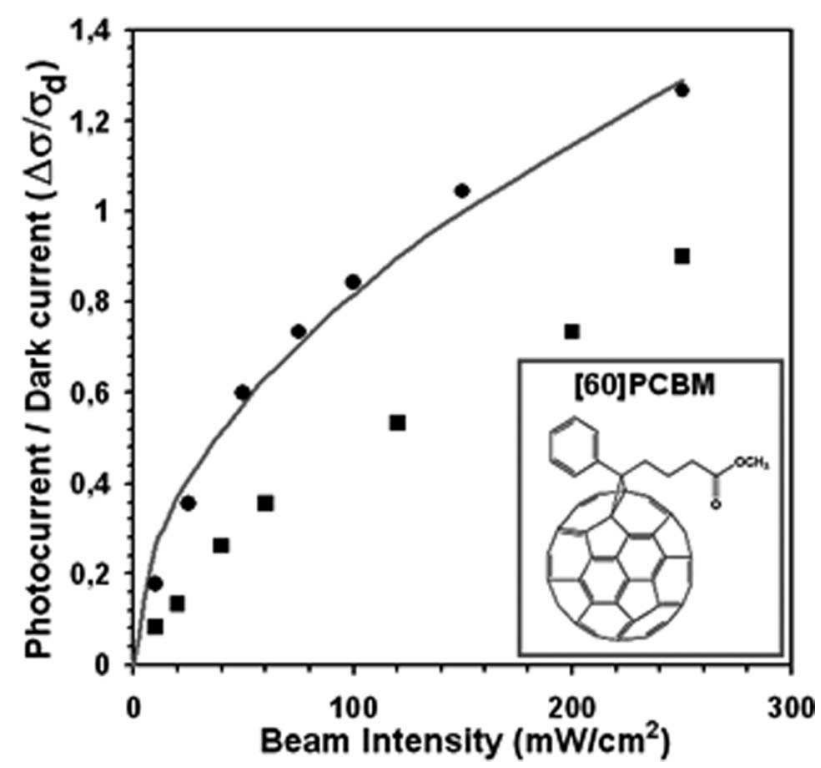

Fig. 1. Photocurrent dependence on light intensity, normalized to the dark current value. M1 (squares), applied field $10 \mathrm{~V} / \mu \mathrm{m}$, dark current density $6 \mu \mathrm{A} / \mathrm{cm}^{2}$. M3 (circles), applied field $0.2 \mathrm{~V} / \mu \mathrm{m}$, dark current density $80 \mathrm{nA} / \mathrm{cm}^{2}$. Continuous line is a fit $\Delta \sigma / \sigma_{D} \propto \sqrt{I}$. Cell thickness equals $20 \mu \mathrm{m}$ for both mixtures. Inset shows molecular structure of [60]PCBM. made of PMMA, E7, and $\mathrm{C}_{60}$ was used. Results on PSLC are presented for a mixture (M1) made up of E7:PMMA:[60]PCBM. For a direct comparison, we prepared a mixture (M2) made up of E7:PMMA: $\mathrm{C}_{60}$ (proportions are given Table 1 ). The $\mathrm{C}_{60}$ concentration was not controlled and corresponded to the maximum solubility achievable at $150^{\circ} \mathrm{C}$. [60]PCBM and $\mathrm{C}_{60}$ were obtained from Nano-C, E7 from Merck, and PMMA with a large molecular weight of $\sim 120 \mathrm{k}$ (120.000) was obtained from Sigma Aldrich. Mixtures are prepared by stirring at $150{ }^{\circ} \mathrm{C}$ until the homogeneous solution was obtained. M2 was transparent at higher temperatures with undissolved particles of $\mathrm{C}_{60}$ at the bottom, whereas $\mathrm{M} 1$ had a homogenous brown color. After cooling to $20^{\circ} \mathrm{C}$ both mixtures solidified, indicating a high influence of polymer network on the macroscopic properties. Thin PSLC films were prepared by filling empty cells with indium tin oxide (ITO)-coated electrodes by capillarity at $150^{\circ} \mathrm{C}$ and cooling them to room temperature. The cell thickness was controlled by spacers of $20 \mu \mathrm{m}$ thickness. To compare with a pure LC case, we prepared a mixture (M3) made up of low molecular weight nematic LC and fullerene. We found that [60]PCBM had a higher solubility in pure LC with respect to $\mathrm{C}_{60}$. The concentration of fullerenes in E7 was limited to about $0.4 \%$. The highest diffraction efficiency among the pure LC mixtures that we prepared was observed for M3, made up of E7:[60] PCBM: $\mathrm{C}_{70}$ (concentrations given Table 1). E7 was obtained from Merck; $\mathrm{C}_{70}$ from Nano-C. The mixture was stirred at $100^{\circ} \mathrm{C}$ before the complete dissolution of fullerenes; after cooling we observed no aggregation of nanoparticles. Higher concentrations of $\mathrm{C}_{70}$ or [60]PCBM were possible at high temperatures, but result in fullerenes clustering at room temperature. M3 was filled by capillarity into cells with homeotropic alignment on both surfaces. The homeotropic alignment was obtained by spin coating with commercial ZLI PA 334 (Merck) according to the product specifications. Mixture behaviors with larger fullerene derivative concentrations (specifically M3) fit well with what is found in the literature $[2,3,8]$. Diffraction efficiency improvements can be well explained by fullerene concentration increases. In next sections, we present the results for a comparison between M3 and M1, to emphasize the role of the polymer network. One of the first observations of this role is the higher concentration of fullerene that we were able to dissolve in PSLC. We attribute it to the phase separated morphology that restricts clustering [60]PCBM.

\section{A. Photoconductivity}

Photoconductivity was measured (dc current) by a model 485 picoampere meter from Keithley. The dark photocurrent was measured under no illumination and the photocurrent was measured by illuminating the sample with an Ar+ laser at $514 \mathrm{~nm}$, with a $8 \mathrm{~mm}$ beam diameter. Results for M1 and M3 are displayed Fig. 1, where the photocurrent/dark current ratio for 
Table 1. Mixture Composition

\begin{tabular}{cccc}
\hline & LC Content & Polymer Content & Fullerene Content \\
\hline M1 & $88 \%$ of E7 & $10 \%$ of PMMA & $2 \%$ of $[60]$ PCBM \\
M2 & $90 \%$ of E7 & $10 \%$ of PMMA & $0.05 \%$ of $\mathrm{C}_{60}$ \\
M3 & $99.6 \%$ of $\mathrm{E} 7$ & $0 \%$ & $0.2 \%$ of $\mathrm{C}_{70}+0.2 \%$ of $[60] \mathrm{PCBM}$ \\
\hline
\end{tabular}

the same sample is displayed. Conductivity under a constant electric field is proportional to the photocurrent density $J=\sigma E$, therefore, the photocurrent/ dark current ratio is $\Delta \sigma / \sigma_{D}$, where $\Delta \sigma$ is the photoconductivity and $\sigma_{D}$ is the dark state conductivity. Photocurrent and diffraction efficiency measurements were performed for M1 with an applied field of $10 \mathrm{~V} / \mu \mathrm{m}$. The dark current value at the same applied voltage and for the surface of $50 \mathrm{~mm}^{2}$ was equal to $3 \mu \mathrm{A}$. The photocurrent for M2 was difficult to measure due to its low value and the high dark current value, probably due to the presence of impurities in commercial products. High photocurrent values compared to M1 indicate an increase in charge generation due to higher fullerene concentration. The dark current and the photocurrent for M3 were measured with an applied electric field of $0.2 \mathrm{~V} / \mu \mathrm{m}$. The dark photocurrent for a surface of $50 \mathrm{~mm}^{2}$ equaled $40 \mathrm{nA}$. Photocurrent increases with an intensity increase, roughly in proportion to the intensity square $\operatorname{root} \Delta \sigma / \sigma_{D} \propto \sqrt{I}$ (Fig. 1 ), in accordance with previous observations [2]. This can be explained by the equilibrium between quadratic recombination of the charges and their generation, through the formation of a complex between the excited photosensitive molecules and the solvent molecules [2].

\section{Results and Discussion}

Photorefractive gratings were recorded with an $\mathrm{Ar}+$ laser at $514 \mathrm{~nm}$; the linearly polarized beam was enlarged to a diameter size of $8 \mathrm{~mm}$ and split into two beams of equal intensity. Polarization of the recording beams was perpendicular to the plane of incidence; it was also perpendicular to the grating wave vector. Diffraction efficiency was measured with a linearly polarized beam of a $\mathrm{He}-\mathrm{Ne}$ laser $(632.8 \mathrm{~nm})$. The intensity of $0.5 \mathrm{~mW}$ on a $1 \mathrm{~mm}$ diameter was lowered so that it cannot influence the grating formation. Relatively large writing beams simplify greatly the experimental setup, as it was easier to have a large recording surface. Absorption of the M3 sample at 514 and $633 \mathrm{~nm}$ was $36 \%$ and $9 \%$, respectively. Samples were set up so that the angle between the sample normal and the bisector of the recording beams was $45^{\circ}$. Diffraction efficiency for a He-Ne laser depends on polarization, reaching a maximum value when oriented parallel to the grating wave vector and decreasing to 0 when perpendicular to it. No diffraction occurred in the absence of the applied dc electric field, or when angles between the bisector of the writing beams and the sample normal approached 0 . These observations are consistent with the orientational photorefractive effect and pure index gratings observed in $[2,10]$. With the same photorefractive grating formation mechanism and a similar chemical composition, the observed difference is attributed to an increase in fullerene concentration in M1 and M3.

Diffraction efficiency is defined as the ratio of the first-order diffraction beam and the incoming beam intensities $\eta=\left(I^{-1} / I\right)$, where scattering and absorption losses have not been subtracted from the incoming intensity. We estimated them to be about $30 \%$ by measuring the ratio of the beam probe intensity before and after the sample, in the absence of writing beams under the applied dc field. Figure 2 shows a typical dependence of the diffraction efficiency on the writing beam intensity for all mixtures. The diffraction intensity reaches saturation for M1 whereas, for $\mathrm{M} 2$, it is far from saturation for the writing intensities used. It should be noted that, in $[9,10]$, a He-Ne laser was used for writing the gratings, with intensities of $4-5 \mathrm{~W} / \mathrm{cm}^{2}$ and similar diffraction efficiencies, whereas for M1 the saturation was obtained for writing intensities of about $20-30 \mathrm{~mW} / \mathrm{cm}^{2}$, corresponding to a two-orders-of-magnitude improvement. For thin gratings, the index modulation can be estimated from $\eta \propto(\Delta n \pi L / \lambda)^{2}$, where $L$ is the grating thickness. Estimates from Fig. 2 give values of $\Delta n \propto 3.5 \times 10^{-3}$, which fits well with the maximum

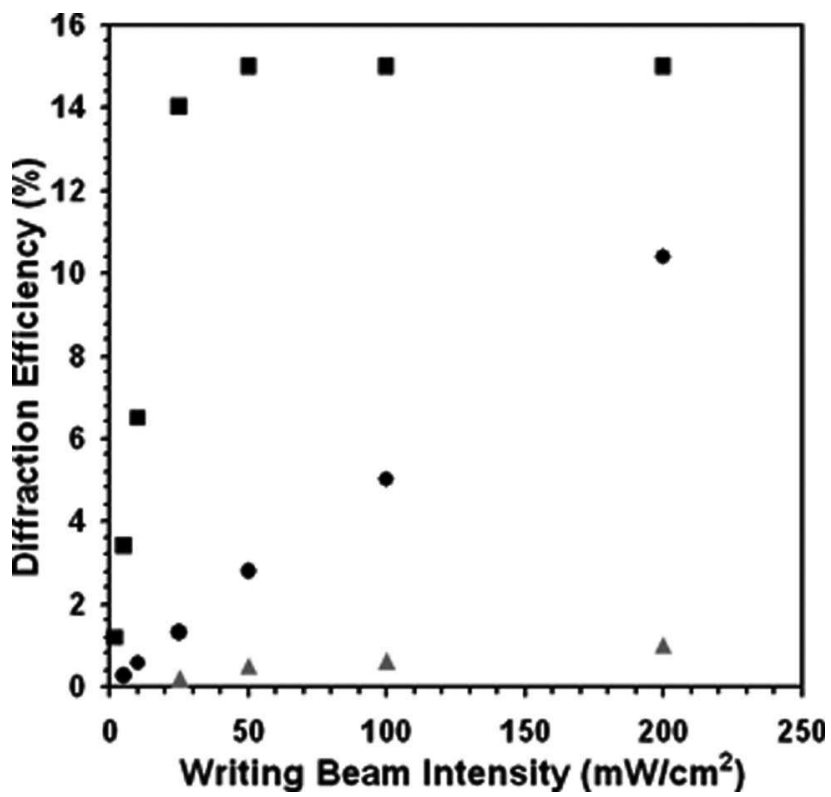

Fig. 2. Probe beam diffraction efficiency dependence on the writing beam intensity sum. M1, squares; M2, triangles; M3, circles. Grating periods for $\mathrm{M} 1=\mathrm{M} 2=8 \mu \mathrm{m}, \mathrm{M} 3=20 \mu \mathrm{m}$; applied field $10 \mathrm{~V} / \mu \mathrm{m}$ for $\mathrm{M} 1$ and $\mathrm{M} 2,0.2 \mathrm{~V} / \mu \mathrm{m}$ for M3. Cell thickness $=20 \mu \mathrm{m}$. (M3, diffraction efficiency at low grating period was too low for accurate measurements.) 
index modulation reported in [10] of $\Delta n \propto 3 \times 10^{-3}$. For M3, we observed a linear increase in diffraction efficiency. Beam intensities used to record the grating are lower than in [3], but an order of magnitude larger than in [8]. The diffraction efficiency for M1 reaches the saturation before $\Delta \sigma / \sigma_{D}$ approaches unity, in contrast to $\mathrm{M} 3$, indicating some influence of the polymer network.

Then we measured the diffraction efficiency dependence on the grating period (Fig. 3). The $50 \mathrm{~mW} / \mathrm{cm}^{2}$ writing beam intensity is larger than the saturation value for the diffraction efficiency of M1 shown in Fig. 2. For M3 at intensities larger than $50 \mathrm{~mW} / \mathrm{cm}^{2}$, higher diffraction efficiencies are observed, but the cutoff grating period remains the same, which is enough to compare with M1. For M1 we observe a diffraction efficiency plateau from 7 to $40 \mu \mathrm{m}$. For M2, the plateau is from 10 to $30 \mu \mathrm{m}$, similar to [10]. For M3 we observe a diffraction efficiency peak at $\Lambda_{x} \approx 40 \mu \mathrm{m}$ with a sharp cutoff at low grating periods and a smoother one at larger periods, which fits well to the theory and experiments [3-8]. The observed diffraction efficiency dependence on grating period for M1 varies from what has been observed earlier for PSLC $[9,10]$; it also differs from observations on pure LC. We clearly observe an improvement in diffraction efficiency at low grating periods, which we attribute to the polymer network.

The counterpart is an increase in writing times to $\sim 20 \mathrm{~s}$ and quite long relaxation times of about $\sim 60 \mathrm{~s}$ for M1. These values should be compared to the recording and relaxation times (in absence of writing beams) that are typically observed in pure LC of $\sim 1 \mathrm{~s}[2,3,8]$ (the existence of fast $\sim 1 \mathrm{~s}$ components was reported in PSLC, also [10]). This behavior indicates the existence of a charge-trapping mechanism

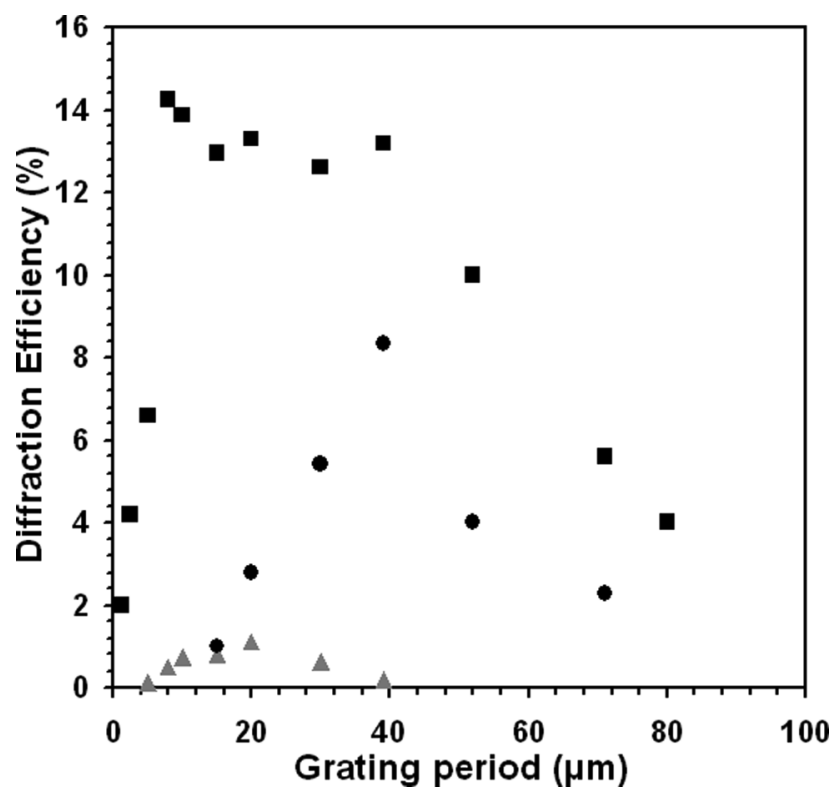

Fig. 3. Probe beam diffraction efficiency dependence on grating period for a $20 \mu \mathrm{m}$ sample. Writing beam intensity $50 \mathrm{~mW} / \mathrm{cm}^{2}$. M1, squares; M2, triangles; M3, circles. either on the interfaces separating the LC domains or in the polymer network. Comparison of photocurrent dependence on light intensity and diffraction efficiency (Fig. 2) shows the polymer network influence, as the saturation in diffraction efficiency occurred a long time before the photocurrent reached the dark current value. According to [2], in the absence of a polymer network, the electric field caused by charge separation can be written as

$$
E_{\mathrm{ph}}=E_{\mathrm{ph}}^{0} \cos (q \xi)=\left(\frac{m k_{B} T}{2 e} q v \frac{\Delta \sigma}{\Delta \sigma+\sigma_{D}}\right) \cos (q \xi) .
$$

$k_{B}$ is the Boltzman constant, $\Delta \sigma$ is the photoconductivity, $\sigma_{D}$ is the dark state conductivity, $v=\left(D^{+}-D^{-}\right) /\left(D^{+}+D^{-}\right)$, with $D^{+}, D^{-}$diffusion speeds of negative and positive ions, and $q$ is the grating wave vector. For PSLC, the liquid crystal is reoriented under the combined effect of the external dc electric field and the periodic electric field $E_{\mathrm{ph}}$. The higher the $E_{\mathrm{ph}}$, the larger the LC director deviation from the external field direction. As noted previously, the writing intensity for which the diffraction efficiency saturation occurs for M1 (as shown in Fig. $3, \sim 30 \mathrm{~mW} / \mathrm{cm}_{\tilde{\tau}}^{2}$ ) is much lower than the intensity at which $\Delta \sigma / \sigma_{d} \tilde{1}, \sim 300 \mathrm{~mW} / \mathrm{cm}^{2}$. This means the maximum of the effective reorienting space-charge field is obtained at lower photocurrent than would be the case for electric fields given by Eq. (1). Such a discrepancy confirms the existence of a trapping mechanism due to the polymer network presence. We can take into account this trapping mechanism, in a phenomenological way, by modifying:

$$
q v \frac{\Delta \sigma}{\left(\Delta \sigma+\sigma_{d}\right)} \rightarrow\left(q v \frac{\Delta \sigma}{\left(\Delta \sigma+\sigma_{d}\right)}+C_{\mathrm{TR}}(t, \Delta \sigma, q)\right)
$$

where $C_{\mathrm{TR}}(t, \Delta \sigma, q)$ corresponds to the trapped charges and depends on the history, similar to the memory effects observed in liquids and polymers. We may rightfully think that the first term reaches a steady-state value much faster than the second. The amount of trapped charges increases with time during the writing process to reach a saturation value, depending on the specific interaction chemistry between LC, polymer, and fullerenes and, in lower degree, to the photocurrent $\Delta \sigma$ and grating period $\Lambda_{x} \propto 1 / q$. When the saturated value of $C_{\mathrm{TR}}(t, \Delta \sigma, q)$ does not depend on $\Delta \sigma, q$, it corresponds to the approximation $C_{\mathrm{TR}}(t, \Delta \sigma, q) \propto C_{\mathrm{TR}}(t)$. If we introduce this approximation into Eq. (2), assuming that $C_{\mathrm{TR}}(\infty)>q v \Delta \sigma /\left(\Delta \sigma+\sigma_{d}\right)$, one would observe a diffraction efficiency plateau, as shown in Figs. 2 and 3 . The introduction of the memory function $C_{\mathrm{TR}}(\bar{t}, \Delta \sigma, q)$ enables us to explain a part of our experimental observations. Accurate computation of the memory function $C_{\mathrm{TR}}(t, \Delta \sigma, q)$ is extremely difficult; even for simple cases of dense liquids, it 
requires a numerical approach or the introduction of uncontrolled approximations.

It is hard to say at this stage what is the exact interaction mechanism between the polymer network and the positive and negative ions. We modified the model proposed in [2] by introducing a phenomenological parameter (memory function $C_{\mathrm{TR}}(t, \Delta \sigma, q)$ ) that can explain that the diffraction efficiency saturates faster than the photocurrent, when one increases the recording beam intensity. It also explains the plateau appearance and the significant diffraction efficiency at small grating periods. In principle, the model proposed in [2] breaks down with the appearance of the trapped charges and one needs to establish a new one. In our opinion, it could be the subject of a separate theoretically oriented article. It would also require additional experiments for identifying what types of charge are getting more easily trapped by the network, as well as the influence of the polymer type on the trapping mechanism. At present, we can only emphasize the critical role of an efficient trapping mechanism for an explanation of the observed experimental data, based on the assumption that the main contribution arises due to the volume effect rather than the surface one observed in $[5,6]$. It is hard to imagine a significant surface contribution in our experiments, as we do not have any particular surface treatment.

The plateaulike behavior of the memory function breaks down for small (less than $5 \mu \mathrm{m}$ ) and large (more than $50 \mu \mathrm{m}$ ) grating periods. At small grating periods the decrease of the space-charge field can be explained by Debye screening, as is proposed in [2]. The screening length calculated there was found to be about $\sim 5 \mu \mathrm{m}$, in agreement with our observations. Moreover, we can rightfully expect that the influence of the polymer network at small grating periods is not very pronounced and the model developed in [2] can be applied to our observations for very small grating periods (less than $5 \mu \mathrm{m}$ ). The slow decrease of the plateau at large grating periods is probably due to a different diffusion regime for the ions, but it is hard to identify a precise mechanism at present.

The charge-trapping mechanism offers a new insight for another way of using the photorefractive effect in PSLC. First, the external electric dc field is applied to PSLC and charges are trapped. Second, the external field is removed and only the spacecharge field operates. In such a case, LC would be reoriented in a larger degree and the index modulation would be larger (Fig. 4). In addition, this improves greatly the PSLC resolution, approaching the resolution of photorefractive polymers with the benefit of smaller applied voltages. It should also be noted that a small residual external field can be necessary if one wants to observe diffraction gratings with the same periods. Otherwise, as the response of the nematic to electric field is quadratic, one would observe the grating with a period twice smaller than the recording one.
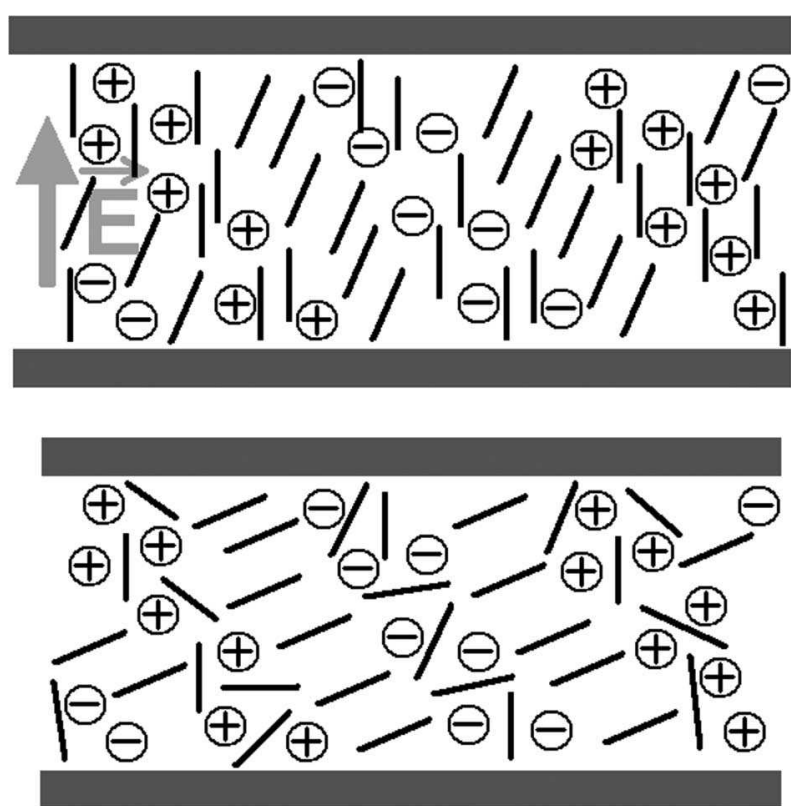

Fig. 4. Phenomenological model for the diffraction efficiency increase mechanism via charge trapping. Increase in refractive index modulation due to higher LC reorientation in the absence of an external dc field (bottom figure). The local field is due to charges trapped in the polymer network.

As can be seen in Fig. 3 , we have recorded a grating with a $\sim 1 \mu \mathrm{m}$ period with a noticeable diffraction efficiency of $\eta \sim 2 \%$. For a transparent PSLC without an external dc field, we could expect a high diffraction efficiency when the dc field is switched off. The proposed approach requires using a transparent PSLC because, in the phase-separated morphology observed for M1 and M2, the composite highly scatters in the absence of an external electric field. Such a transparent PSLC can be made by using a technique described in $[14,15]$, where the polymer used for stabilization has a mesogenic side chain. Therefore, the polymer and the LC can form an oriented transparent phase. Another possibility is using a mesogenic monomer and polymerize it in a nematic LC phase.

To test this idea, we realized a transparent PSLC by mixing a nematic LC, 5CB; a mesogenic monomer, RM257; a photoinitiator, Igracure 651; and a photosynthesizer, [60]PCBM, with proportions of 89.4:10:0.5:0.1. The mixture was filled into a $20 \mu \mathrm{m}$ cell and UV cured at $50{ }^{\circ} \mathrm{C}$ with $10 \mathrm{~mW} / \mathrm{cm}^{2}$ intensity. RM257 is a mesogenic diacrylate commonly used to stabilize polymer. After curing it forms a branched polymer network, preserving the LC orientation. The charge-generation process remains the same as for M1 and is due to the interaction between LC and fullerene. Other ways of preparing transparent PSLC are possible, such as is described in $[14,15]$. The important point is the presence of the charge-trapping mechanism in the material bulk due to the presence of the polymer network.

To confirm the presence of the memory effect in this compound, we recorded a grating at a $\Lambda_{x}=2.5 \mu \mathrm{m}$ period under a $5 \mathrm{~V} / \mu \mathrm{m}$ applied electric 
field and a writing power of $50 \mathrm{~mW} / \mathrm{cm}^{2}$. During the recording no diffraction of the $\mathrm{He}-\mathrm{Ne}$ probe beam was observed. After $30 \mathrm{~s}$ we removed the writing beam and the applied dc field. We observed a slowly decaying grating with $4 \%$ efficiency. It confirmed the proposed operating method and the existence of a trapping mechanism in some PSLC. This mechanism operates differently in this mixture, as we do not observe a noticeable diffraction during the writing stage. This new material has a complex behavior with respect to the grating period and applied electric field, which will require further investigations.

A similar effect of an increase in diffraction efficiency after decreasing the applied external field was observed in [17]. They observed a similar memory effect, when the local space-charge field decreased more slowly than the external field, so that the relative contribution of the photogenerated space-charge field could increase during a certain time period. However, the polymer matrix in [17] is a conductive one, so that the trapping mechanism is different.

\section{Conclusion}

We found a fullerene derivative [60]PCBM soluble in high concentration in a polymer-LC composite, made of PMMA and E7. Solubility up to $0.5 \%$ was observed in pure LC, as well. An increase in fullerene concentration, operating as an efficient charge generator, resulted in an increase in diffraction efficiency. We obtained PSLC efficiency improvements with respect to previous work by a factor of 10-100, depending on the writing intensity and grating periods. High diffraction efficiency improvements have been observed in particular at small grating periods with respect to both LC and similar polymer-LC composites investigated previously. A part of the improvement could be attributed to an increase in charge generation, similar to that observed earlier in pure LC [8]. Another part was attributed to the trapping mechanism due to the polymer network. We proposed a phenomenological description of the trapping mechanism by introducing a memory function that describes the interaction of the photogenerated ions and the polymer network. On this basis, a new approach for designing high-efficiency polymerLC composites has been proposed and is currently being investigated. The idea is to record the grating by trapping the charges in the bulk, via the polymer network, in the presence of an external electric field and to read it without an external applied field. In the absence of external electric fields, the media exhibits a higher index modulation, resulting in a higher diffraction efficiency, especially at small grating periods.

The authors thank L. Dupont and P. Gravey for valuable discussions about the manuscript. This project is granted by the Region Bretagne under the project FSE-Convention $n^{\circ} 1860$.

\section{References}

1. I. C. Khoo, H. Li, and Y. Liang, "Observation of orientational photorefractive effects in nematic liquid crystals," Opt. Lett. 19, 1723-1725 (1994).

2. E. V. Rudenko and A. V. Sukhov, "Photoinduced electrical conductivity and photorefraction in a nematic liquid crystal," JETP Lett. 59, 142-146 (1994).

3. I. C. Khoo, "Holographic grating formation in dye- and fullerene $\mathrm{C}_{60}$-doped nematic liquid-crystal film," Opt. Lett. 20, 2137-2139 (1995).

4. G. P. Wiederrecht, B. A. Yoon, and M. R. Wasielewski, "High photorefractive gain in nematic liquid crystals doped with electron donor and acceptor molecules," Science 270, 17941797 (1995).

5. P. Pagliusi and G. Cipparrone, "Surface-induced photorefractive-like effect in pure liquid crystals," Appl. Phys. Lett. 80, 168-170 (2002).

6. J. Zhang, V. Ostroverkhov, K. D. Singer, V. Reshetnyak, and Yu. Reznikov, "Electrically controlled surface diffraction gratings in nematic liquid crystals," Opt. Lett. 25, 414-416 (2000).

7. W. Lee and C.-S. Chiu, "Observation of self-diffraction by gratings in nematic liquid crystals doped with carbon nanotubes," Opt. Lett. 26, 521-523 (2001).

8. I. C. Khoo, S. Slussarenko, B. D. Guenther, Min-Yi Shih, P. Chen, and W. V. Wood, "Optically induced space-charge fields, dc voltage, and extraordinarily large nonlinearity in dye-doped nematic liquid crystals," Opt. Lett. 23, 253-255 (1998).

9. H. Ono and N. Kawatsuki, "Orientational photorefractive effects observed in polymer-dispersed liquid crystals," Opt. Lett. 22, 1144-1146 (1997)

10. H. Ono, H. Shimokawaa, A. Emotoa, N. Kawatsukib, "Effects of droplet size on photorefractive properties of polymer dispersed liquid crystals," Polymer 44, 7971-7978 (2003).

11. A. Golemme, B. L. Volodin, B. Kippelen, and N. Peyghambarian, "Photorefractive polymer-dispersed liquid crystals," Opt. Lett. 22, 1226-1228 (1997).

12. J. G. Winiarz and P. N. Prasad, "Photorefractive inorganic organic polymer-dispersed liquid-crystal nanocomposite photosensitized with cadmium sulfide quantum dots," Opt. Lett. 27, 1330-1332 (2002).

13. G. P. Wiederrecht, "Photorefractive liquid crystals," Annu. Rev. Mater. Res. 31, 139-169 (2001).

14. H. Ono, A. Hanazawa, T. Kawamura, H. Norisada, and N. Kawatsuki, "Response characteristics of high-performance photorefractive mesogenic composites,” J. Appl. Phys. 86, 1785-1790 (1999).

15. N. Kawatsuki, H. Norisada, T. Yamamoto, H. Ono, and A. Emoto, "Photorefractivity in polymer dissolved liquid crystal composites composed of low-molecular-weight nematic liquid crystals and copolymer comprising mesogenic side groups," Sci. Tech. Adv. Mater. 6, 158-164 (2005).

16. J. C. Hummelen, G. Yu, J. Gao, F. Wudl, and A. J. Heeger, "Polymer photovoltaic cells: enhanced efficiencies via a network of internal donor-acceptor heterojunctions," Science 270, 1789-1791 (1995).

17. A. Golemme, B. Kippelin, and N. Peyghambarian, "On the mechanism of orientational photorefractivity in polymer dispersed nematics," Chem. Phys. Lett. 319, 655-660 (2000). 